\title{
Preparation and characterization of nanomaterials based on bifacial carbon nanotubes and iron oxides: Application in catalysis
}

\author{
A. Zafour-Hadj-Ziane ${ }^{1, a}$, M. Djiab ${ }^{1}$ and E. Flahaut ${ }^{2}$ \\ 1 Laboratoire de Génie Chimique Université Saad Dahlab de Blida, Route de Soumaa, BP. 270, Algérie \\ 2 CIRIMAT, Université Paul Sabatier, Toulouse, France
}

\begin{abstract}
The application of magnetic particles technology for the development of new nanomaterials has received considerable attention in recent years. In this context, the objective of this study is firstly, to prepare new catalytic materials that gather the strong adsorption capacities of carbon nanotubes and magnetic properties of iron, it concerns nanocomposites based on a mixture of carbon nanotubes in a very small amounts and iron oxide. Secondly we want to appear their capacities in catalytic oxidation reactions of phenol. Synthesis under the optimal conditions was carried out at different $\mathrm{pH}$. And the characterization of this new nanomaterial reveals a good specific surface area BET, the identification of carbon nanotubes within the matrix was performed by infrared spectroscopy and transmission electron microscopy. The use of this new material as a catalytic support in catalytic oxidation reactions of phenol indicates the high selectivity of this latter and a yield better than this obtained with iron oxide supported by activated carbon. The good catalyst regeneration of the new catalysis and the improvement in their properties are the interesting parameters for the new type nanomaterials.
\end{abstract}

\section{INTRODUCTION}

Since the discovery of carbon nanotubes by Lijima in 1991 [1], research has focused on these new nanomaterials known by their electronic, mechanical and thermal properties. [2], The introduction of carbon nanotubes to polymers and other organic and inorganic molecules can acquire new properties for several applications such as microelectronics, energy storage, rechargeable batteries etc. ....

Recently, a new generation of materials for catalysis have been developed by insertion of new compounds with additional properties such as metallic groups at magnetic character [3], In this context, several metals were used such as $\mathrm{Ni}, \mathrm{Cu}$ [4], $\mathrm{Pt}$ [5], $\mathrm{Fe}$ and $\mathrm{Co}$ [6], and particles oxides of $\mathrm{SiO} 2$ [7], $\mathrm{SnO} 2$ [8], $\mathrm{Al} 2 \mathrm{O} 3$ [9] and $\mathrm{TiO} 2$ [10], which are deposited on the surface of nanotubes.

Several studies have shown that the use of iron oxide supported on carbon nanotubes as catalyst present an industrial and environmental interest.

On the other hand, aqueous effluents contaminated by organic matter from the chemical industry and agricultural activity, are less biodegradable because of the high concentration of pollutant (COD of $500 \mathrm{mg}$ to $1500 \mathrm{mg} / \mathrm{L}$ ). The harmful effects of pollution by phenols lead to degradation of fauna; flora and fish, especially species at patty chain (eel, salmon, trout .....) accumulate phenols and may contain more than $30 \mathrm{mg} / \mathrm{kg}$, then are so inedible. Oxidation processes in the liquid phase seems to offer an effective and less expensive solutions. On this principle, Alejandre and al $[11,12]$ studied the catalytic activity of copper and nickel in the oxidation reaction of phenol in aqueous medium, they showed that these catalysts are highly active in oxidation. The conversion rate of phenol depends on the catalyst preparation and the oxidation reaction is very selective in $\mathrm{CO} 2$. Catalysts based

a e-mail: amelzafour@yahoo.fr on heavy metal supported onto clays (montmorillonite) [13], silica [14] showed a high catalytic activity in oxidation of phenol by hydrogen peroxide. At atmospheric pressure and temperature of to $80^{\circ} \mathrm{C}$, phenol degradation is fast $(15 \mathrm{~min})$ and total with a mineralization selectivity of $93-59 \%$. Original study on the treatment of a phenolic solution on heterogeneous phase by hydrogen peroxide with Fe-ZSM-5 catalyst was carried out by Fajerwerg and al [15]. At $90^{\circ} \mathrm{C}$ under atmospheric pressure a total elimination of phenol was recorded.

\section{MATERIALS AND METHODS}

Nanotubes of carbon are synthesis in the Carbon nanotubes: were synthesized at interuniversity Center for Materials research and engineering in Toulouse by the process Catalytic Chemical Vapor Deposition, after extraction, they are stored in wet form.

\subsection{Preparation of nanocomposites}

The preparation of materials DWCNT/ iron oxide was carried out as follows: A mixed solution of $0.1 \mathrm{M}$ hexahydrate ferric chloride and $0.05 \mathrm{M}$ tetrahydrate ferrous chloride with a molar ratio equal to $1 / 2$. Different amounts of carbon nanotubes biface were added to the previous solution. At a temperature of $70^{\circ} \mathrm{C}$ and at constant perimeter, a solution of $\mathrm{NH} 4 \mathrm{OH} 5 \mathrm{M}$ was introduced dropwise to precipitate iron oxides.

\subsection{Preparation of catalysts}

All catalysts were prepared by impregnation. The impregnation solution $\mathrm{FeCl} 3.6 \mathrm{H} 2 \mathrm{O}$ (volume equal to the pore volume: $2 \mathrm{ml}$ ) was added drop by drop to 


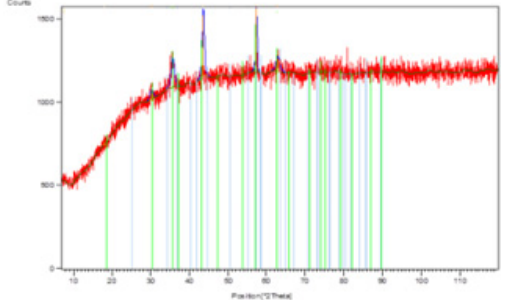

Figure 1. Spectrum DRX of DWCNT/iron oxyde.

the support. The concentration of the solution was calculated in condition that the carbon supports are loaded by $10 \%$ and $20 \%$ of metal.

\section{CHARACTERIZATION OF NANOMATERIALS}

\subsection{Analyse with DRX}

The XRD spectrum obtained is comparable to the spectrum obtained by Xianjia Peng and al [15], the peaks correspond to different crystal structures of iron oxide and CNT. The peaks corresponding to diffraction angles $2 \theta=43.56^{\circ}$ and $57.27^{\circ}$ are assigned to the structure of the double walled carbon nanotubes. The peaks corresponding to diffraction angles $2 \theta=35.65^{\circ} 62.97^{\circ}$ and $30.18^{\circ}$ are assigned to different types of iron oxides: Magnetite $\left(\mathrm{F}_{3} \mathrm{O}_{4}\right)$ and

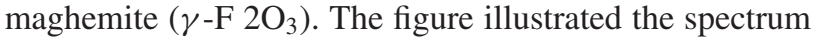
DRX of DWCNT/iron oxyde.

\subsection{Specific surface measurement}

Specific surface of oxide powders was performed with an automatic Micromeritics Flow Sorb II 2300, by nitrogen adsorption at the temperature of liquid nitrogen. Samples were degassed for 1 hour at $120^{\circ} \mathrm{C}$.

\subsection{Transmission electron microscopy}

The powders were dispersed to be observed by ultrasound (tank) in ethanol. Few drops of the suspension were deposited on a grid of copper sample holder with a carbon film pierced with holes (Lacey). CNTs are observed at the holes in order to avoid interference with the carbon film.

\subsection{Magnetic properties of CNT/iron oxyde}

We determined the Curie constants at different temperatures using the following formula

$$
\chi P=C / \mathrm{T}
$$

$\chi P$ is the paramagnetic susceptibility, $\mathrm{C}$ the Curie constant and $\mathrm{T}$; Temperature in ${ }^{\circ} \mathrm{C}$.

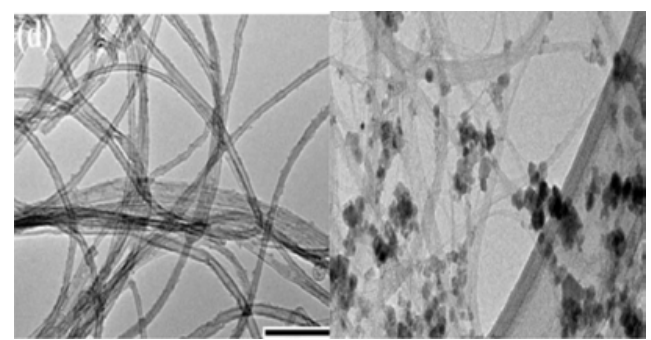

Figure 2. Micrographes TEM at $50 \mathrm{~nm}$ a) crude DWCNT b) DWCNT/iron oxide.

\section{OXIDATION REACTION OF PHENOL}

\subsection{Experimental procedure}

The reaction of phenol oxidation is performed in batch system. For a volume of $100 \mathrm{ml}$ of a $5.10^{-4} \mathrm{M}$ phenol solution, we added a known quantity of washed catalyst. The beginning of oxidation reaction under standard conditions (C.N.T.P) correspond to the added of oxidizing agent $\left(\mathrm{H}_{2} \mathrm{O}_{2} 5 \mathrm{ml}\right.$ of $\left.10^{-3} \mathrm{M}\right)$. Samples were taken at known interval time in order to follow the evolution of $\mathrm{pH}$,oxidant concentration, phenol.

\subsection{Analytical techniques}

The determination of hydrogen peroxide in stock solutions having concentrations up to $10^{3} \mathrm{M}$, released using the iodometric method. For concentrations between $10^{-5} \mathrm{M}$ and $10^{-3} \mathrm{M}$, Eisenberg method is used [14]. The quantification of Iron is performed by atomique absorption. The derivate products are analyzed by liquid chromatography HPLC high pressure.

\section{RESULTS AND DISCUSSION}

\subsection{Characterization of the nanocomposites}

- Analysis by Transmission Electron Microscopy:

The following figures show the TEM pictures taken at two different magnifications, we see clearly the globules of iron oxide fully inserted between the wires of nanotubes at different magnifications.

Moreover, Iron oxides cover well the nanotube surface throughout its length, this is confirmed in reference [17].

In the figure a, we see clearly the wires of bifacial nanotubes in the absence of iron oxide.

\section{- Specific surface area}

The specific surface area was estimated at $259.91 \mathrm{~m}^{2} / \mathrm{g}$ while the pure CNTs have a specific surface of $335.5 \mathrm{~m}^{2} / \mathrm{g}$, the introduction of CNTs to iron oxides used to prepare materials with specific surface areas fairly significant.

\section{- Magnetic properties}

The Curie constant determined at $200{ }^{\circ} \mathrm{C}$ showed that it remains constant with a value of 4.43 and 4.41 before and after reaction of oxidation respectively.

The iron chloride in the water gives $\mathrm{Cl}^{-}$whose electronic structure without magnetic moment. $\mathrm{Fe} 3+$ ions 
have five electrons in the layer $3 \mathrm{~d}$, then a layer incomplete and a nonzero magnetic moment and behave like the molecules of an ideal gas and therefore fall within the framework of the theory which neglects the interaction between the magnetic moments.

Finally, that nanomaterials retain their properties after use in catalysis, however this study are preliminary and must be considered part of a more rigorous with large scale of temperature.

\subsection{Oxidation reaction}

The purpose of this reaction is just to test the catalytic activity of synthesized nanocomposites.

The derivate products of the oxidation were analyzed by HPLC. The results are shown in the figure below.

Toward these results we observed that the phenol was oxidized to $99 \%$ in $60 \mathrm{~min}$, the kinetics is very fast in the first minutes, and then stabilizes to reach equilibrium state after one hour. The amount of the consumed $\mathrm{H}_{2} \mathrm{O}_{2}$ varies in a manner proportional to the amount of phenol oxidized until the complete exhaustion of the oxidizing agent. HPLC analysis showed that there is formation of several derivates products; the final product of the oxidation is oxalic acid. In order to confirm the obtained results in the presence of solid catalyst in aqueous media and show clearly that oxidation was not due to the release of iron in the solution, a catalytic blank test is performed in homogeneous phase, in the same conditions as in the presence of the oxidation catalyst. $1 \mathrm{mg}$ of iron is introduced in the form of ferric iron nitrate.

The results show that the rate of phenol eliminated in homogeneous oxidation does not exceed $20 \%$. Finally, we can conclude that the obtained results in the presence of solid catalyst show that the oxidation reaction happens mainly in heterogeneous phase and this because of the stability and activity of the catalysts.
The study of the influence of the amount of iron in the nanocomposite on the yield of the reaction would be interesting.

\section{CONCLUSION}

Carbon nanotubes could confer to iron oxides very interesting properties as a catalyst with high activity, this is confirmed by the strong performance of oxidation and removal of phenol which is almost complete. The amount of CNT introduced is very small, which seems very interesting from an economic perspective. Several parameters are studied to prove the efficiency of such catalysts enhanced by carbon nanotubes. Finally, it is concluded from the environmental point of view, phenol oxidation is the most effective method for its removal and this kind of new materials is very promising since it can be easily regenerated and re-used by removing phenol through appropriate techniques.

\section{References}

[1] Iijima S. Helical microtubules of graphitic carbon. Nature, 1991, 354: 56-58.

[2] Shang H.Y., Liu C.G., Xu Y.Q., et al. Effect of the surface modification of multi-walled carbon nanotubes (MWCNTs)on hydrodesulfurization activity of Co-Mo/ MWCNTs catalysts. New Carbon Materials, 2004, 19(2): 131-136.

[3] Luo J.Z., Gao L.Z., Leung Y.L., et al. The decomposition of NO on CNTs and $1 \mathrm{wt} \% \mathrm{Rh} / \mathrm{CNTs}$. Catal Lett, 2000, 66 (1-2): 91-97.

[4] Ang L.M., Hor T.S.A., Xu G.Q., et al. Decoration of activated carbon nanotubes with copper and nickel. Carbon, 2000, 38(3): 363-372.

[5] Ledoux M.J., Vieira R., Pham-Huu C., et al. New catalytic phenomena on nanostructured (fibers and tubes) catalysts. J Catal, 2003, 216 (1-2): 333-342. 